\title{
Lipidmessung muss nicht nüchtern erfolgen
}

\begin{abstract}
Am Morgen nichts essen zu dürfen, empfinden viele Patienten als unangenehm. Für eine routinemäßige Lipidbestimmung ist das Nüchternbleiben nun nicht mehr erforderlich.
\end{abstract}

Für eine routinemäßige Lipidbestimmung müssen Patienten nicht mehr nüchtern in der Praxis erscheinen - diese Auffassung wird nun von internationalen Experten der European Atherosclerosis Society (EAS) und der European Federation of Clinical Chemistry and Laboratory Medicine (EFLM) in einem gemeinsamen Konsensuspapier vertreten. Nur in speziellen Situationen oder bei hohen Triglyzeridspiegeln im Plasma von $>5 \mathrm{mmol} / \mathrm{l}$ kann demnach eine Nüchtern-Blutabnahme noch erforderlich werden.

\section{Schnelle Entscheidungsfindung}

Für Patienten, Ärzte und Laborpersonal bedeute die Lipidmessung im nicht-nüchternen Zustand eine große Erleichterung, die letztlich auch die Compliance der $\mathrm{Pa}$ tienten verbessern könne, argumentieren die Autoren des Konsensuspapiers um Børge Nordestgaard, Kopenhagen. Denn gerade für Kinder, Diabetiker und ältere Menschen kann das Nüchternbleiben eine Belastung darstellen. Zudem entfällt in vielen Fällen die Notwendigkeit, noch einmal in die Praxis zu kommen, nur um die Lipidwerte nüchtern messen zu lassen. Und den Ärzten ermöglicht die sofortige Messung eine schnellere Entscheidungsfindung.

Generelle Bedenken gegen eine Lipidbestimmung im nicht-nüchternen Zustand sind eine nicht ausreichende Messgenauigkeit und das Fehlen von evaluierten Referenzwerten. Nordestgaard und Kollegen zufolge gibt es mittlerweile aber Daten aus aktuellen Kohorten- und Registerstudien, die belegen, dass eine Nahrungsaufnahme das Lipidprofil nur geringfügig beeinflusst und die zu beobachteten Veränderungen der Lipidkonzentrationen sich klinisch nicht signifikant auswirken. Darüber hinaus zeigen zahlreiche Studien, dass nicht-nüchtern gemessene Lipidwerte das kardiovaskuläre Risiko ähnlich gut oder besser widerspiegeln.

\section{Nüchtern nur manchmal sinnvoll}

Die Lipidmessung im nüchternen Zustand sollte nach Nordestgaard und Kollegen aber nicht völlig abgeschrieben werden. Beide Messmethoden sollten sich gegenseitig nicht ausschließen. Generell sollte man für die Entscheidung, ob nüchtern oder nicht-nüchtern gemessen wird, den Sinn und Zweck der Lipidmessung berücksichtigen. Wenn es etwa um die Diagnose einer genetisch bedingten Dyslipidämie gehe, habe die Nüchtern-Blutabnahme ihren berechtigten Stellenwert, führen die Experten aus, während sie im Falle der ersten Screeningstufe weniger wichtig sei.

In folgenden Situationen kann dem Konsensuspapier zufolge eine NüchternBlutabnahme zur Lipidbestimmung erforderlich werden:

- Triglyzeridwert $>5 \mathrm{mmol} / \mathrm{l}$ (440 mg/d) bei Nicht-Nüchtern-Blutabnahme,

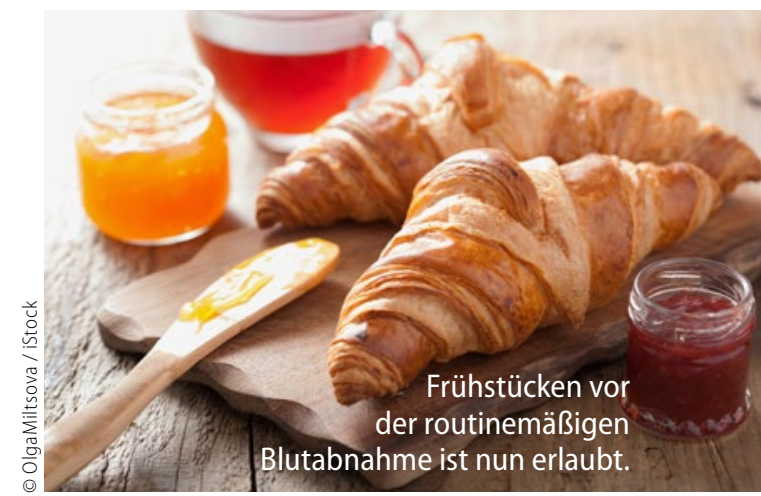

- geplanter Therapiebeginn mit Medikamenten wie Steroiden, Östrogenen oder Retinsäure,

- Verlaufskontrolle einer Hypertriglyzeridämie-induzierten Pankreatitis,

- bekannte Hypertriglyzeridämie mit anschließender Überweisung in eine Lipidklinik,

- falls noch weitere Bluttests nötig sind, die nüchtern erfolgen müssen.

Alle anderen Patienten müssten bei der Blutabnahme nicht nüchtern sein.

Sinnvoll wäre es, die Patienten anzuhalten, am Tag der Lipidbestimmung keine fettigen Mahlzeiten zu sich zu nehmen und vor der Messung nichts zu trinken, da eine Flüssigkeitszufuhr die Werte verfälschen kann. Patienten mit lebensbedrohlichen Messwerten sollte man zu einem Spezialisten überweisen.

(vSC)

European Heart J 2016, online 26. April

\section{RAS-Hemmer, Antialdosteron \& Co.}

\section{Herzschwäche: Behalten Sie die Nieren im Blick!}

Etwa 40-50\% aller Patienten mit Herzinsuffizienz haben erhöhte Kreatininwerte. Und die haben Folgen.

Prof. Dr. Christoph Wanner aus Würzburg berichtete beim DGIM-Kongress 2016 über schwedische Registerdaten. Seine eindrucksvolle Botschaft: Je kaputter die Niere, desto höher die Sterblichkeit bei Herzinsuffizienz. Umgekehrt haben herzinsuffiziente Patienten gute Karten, solange die Niere intakt ist. Deshalb sollte zuerst der Blutdruck unter Einsatz eines RAS-Hemmstoffs auf unter 130/80 mmHg eingestellt werden, so Wanner. Ein zusätzliches Antialdosteron ist auch bei eingeschränkter Nierenfunktion zu erwägen. Insbesondere bei diabetischer Nephropathie gibt es Hinweise, dass die Nierenfibrose dadurch verzögert werde.

Was die Diurese angeht, hat Wanner eine klare Empfehlung: „Furosemid sollte vermieden werden, da es den Sympathikus aktiviert und zu Diuretikaresistenz führt." Ist ein Schleifendiuretikum nötig, sei Torasemid die bessere Wahl. (gvg)

DGIM-Kongress, 2016, 9.-12. April, Mannheim 